\title{
Paraxial optics of charged particles in solenoids
}

\author{
Timofey Gorlov@* \\ Oak Ridge National Laboratory, Oak Ridge, Tennessee 37831, USA
}

(Received 28 October 2019; accepted 11 February 2020; published 10 March 2020)

\begin{abstract}
Paraxial optics of charged particles in solenoid magnets with axisymmetric field is considered. Analytic and symplectic transverse maps for charged particle motion through solenoids with 3D magnetic fields have been derived. The formula for transverse maps contains both linear and nonlinear effects caused by entrance and exit fringe fields of the solenoid. The explicit transfer map can be used for applications of accelerator physics that use solenoid magnets.
\end{abstract}

DOI: 10.1103/PhysRevAccelBeams.23.034001

\section{INTRODUCTION}

We derived an analytic nonlinear-linear transverse map for single charged particle dynamics through solenoid magnets in a paraxial approximation. Solenoid magnets have been used in electron optics with relatively small energies of electrons [1]. Also, solenoids have wide application in high energy ion accelerators and colliders [2-5]. Recently, there is particular interest in using the solenoids for integrable optics $[6,7]$ and for self-consistent beams [8].

In accelerator physics, for calculation of single particle dynamics in solenoids, the most commonly used solution in the form of a transfer map can be found, for example in [1]. This transfer map is a good linear approximation of beam dynamics but it considers only the linear effects and does not take into account the detailed distribution of 3D magnetic field in the solenoid. In modern physics, precise linear and nonlinear beam dynamics calculations are performed by using numerical simulations of particles in given 3D fields $[9,10]$. An analytic approach for nonlinear beam dynamics in solenoids has been performed in [11]. Simple and effective nonlinear kick estimate can be performed by using analytic integration of motion equations like in $[12,13]$.

In this paper we derive an analytic nonlinear transfer map for beam dynamics in solenoids directly from Newton's force equation for charged particle motion in a 3D magnetic field. For solving the equation, we developed and applied a method of iterations that was initially used in [14] for solving a quadrupole magnet. The method was originally applied to find the second order solution in magnetic field.

\footnotetext{
tg4@ornl.gov
}

Published by the American Physical Society under the terms of the Creative Commons Attribution 4.0 International license. Further distribution of this work must maintain attribution to the author(s) and the published article's title, journal citation, and DOI.
Here we developed this method to find the $N$ th order solution that depends on parameter $N$ in explicit analytic form. Then by calculating $N \rightarrow \infty$ we found precise analytic solution (with some limitations) for particle dynamics. The solution has been represented in the form of transfer maps widely used in accelerator physics.

\section{PARAXIAL MOTION IN AXIAL-SYMMETRIC MAGNETIC FIELDS}

We use Newton's relativistic force equation for motion of a charged particle in a static magnetic field:

$$
\ddot{\mathbf{r}}=\frac{q}{m \gamma} \dot{\mathbf{r}} \times \mathbf{B} .
$$

The axially symmetric static magnetic field in Cartesian coordinates has the following form:

$$
\mathbf{B}=\left(\begin{array}{c}
\sum_{i=0}^{\infty} \frac{(-1)^{i+1} x\left(x^{2}+y^{2}\right)^{i}}{2^{2 i+1} i !(i+1) !} \frac{\partial^{2 i+1} B(z)}{\partial z^{2 i+1}} \\
\sum_{i=0}^{\infty} \frac{(-1)^{i+1} y\left(x^{2}+y^{2}\right)^{i}}{2^{2 i+1} i !(i+1) !} \frac{\partial^{2 i+1} B(z)}{\partial z^{i+1}} \\
\sum_{i=0}^{\infty} \frac{(-1)^{i}\left(x^{2}+y^{2}\right)^{i}}{4^{i}(i !)^{2}} \frac{\partial^{2 i} B(z)}{\partial z^{2 i}}
\end{array}\right)
$$

where $B(z)$ is the distribution of the $z$-component of the B-field along the $z$ axis. We now truncate magnetic field (2) for calculation of particle dynamics near z-axis:

$$
\mathbf{B}=\left(\begin{array}{c}
-\frac{1}{2} \frac{\partial B}{\partial z} x+\frac{1}{16} x\left(x^{2}+y^{2}\right) \frac{\partial^{3} B}{\partial z^{3}} \\
-\frac{1}{2} \frac{\partial B}{\partial z} y+\frac{1}{16} y\left(x^{2}+y^{2}\right) \frac{\partial^{3} B}{\partial z^{3}} \\
B-\frac{1}{4}\left(x^{2}+y^{2}\right) \frac{\partial^{2} B}{\partial z^{2}}
\end{array}\right) .
$$

Substituting (3) into (1) we obtain a 3D time-dependent system of equations for particle motion in a 3D magnetic field: 


$$
\begin{aligned}
& \ddot{x}=+\frac{q}{m \gamma}\left[B \dot{y}+\frac{y}{2} \frac{\partial B}{\partial z} \dot{z}-\frac{x^{2}+y^{2}}{16}\left(y \dot{z} \frac{\partial^{3} B}{\partial z^{3}}+4 \dot{y} \frac{\partial^{2} B}{\partial z^{2}}\right)\right] \\
& \ddot{y}=-\frac{q}{m \gamma}\left[B \dot{x}+\frac{x}{2} \frac{\partial B}{\partial z} \dot{z}-\frac{x^{2}+y^{2}}{16}\left(x \dot{z} \frac{\partial^{3} B}{\partial z^{3}}+4 \dot{x} \frac{\partial^{2} B}{\partial z^{2}}\right)\right] \\
& \ddot{z}=\frac{q}{m \gamma}(y \dot{x}-x \dot{y})\left(\frac{x^{2}+y^{2}}{16} \frac{\partial^{3} B}{\partial z^{3}}-\frac{1}{2} \frac{\partial B}{\partial z}\right)
\end{aligned}
$$

where the particle vector $\mathbf{r}=\{x, y, z\} \equiv\{x(t), y(t), z(t)\}$ depends on time.

Equations (4) can be rewritten using a paraxial approximation that is frequently used in accelerator physics in the case of high longitudinal particle momentum. In this approximation $\dot{y}, \dot{x} \ll \dot{z}=v=\beta c=$ const. In the general case, transformation of $\ddot{x}$ and $\ddot{y}$ in (4): $\ddot{\mathbf{r}} \rightarrow \mathbf{r}^{\prime \prime}(z) \dot{z}^{2}+\mathbf{r}^{\prime}(z) \ddot{z}$ produces term $\mathbf{r}^{\prime}(z) \ddot{z}$ with the second order angular aberrations $x^{\prime 2}, y^{\prime 2}, x^{\prime} y^{\prime}$. For typical accelerator values $r^{\prime}<50 \mathrm{mrad}$, the relative angular nonlinear aberration is much smaller than spatial one $r^{\prime} /(r / R) \ll 1$ where $R$ is an aperture of solenoid. In this paper we omit this term for simplicity of final solution. In the end we will perform benchmark that compares approximation from (4) to (5). Substituting $\ddot{\mathbf{r}} \rightarrow \mathbf{r}^{\prime \prime}(z) \beta^{2} c^{2}$ and a new variable $z=\beta c t$ into (4) we obtain 2D equations for transverse motion of a particle in a 3D magnetic field:

$$
\begin{aligned}
\frac{d^{2} x}{d z^{2}} & =+k\left[b \frac{d y}{d z}+\frac{y}{2} \frac{d b}{d z}-\frac{x^{2}+y^{2}}{16}\left(y \frac{d^{3} b}{d z^{3}}+4 \frac{d y}{d z} \frac{d^{2} b}{d z^{2}}\right)\right] \\
\frac{d^{2} y}{d z^{2}} & =-k\left[b \frac{d x}{d z}+\frac{x}{2} \frac{d b}{d z}-\frac{x^{2}+y^{2}}{16}\left(x \frac{d^{3} b}{d z^{3}}+4 \frac{d x}{d z} \frac{d^{2} b}{d z^{2}}\right)\right] \\
k & =\frac{q B_{0}}{m c \beta \gamma} .
\end{aligned}
$$

Here, we introduced a parameter $k$ and the "normalized" longitudinal function $b \equiv b(z)=B(z) / B_{0}$ where $B_{0}=$ $B\left(z_{\max }\right)$ is the amplitude of the magnetic field in solenoid (see Fig. 1).

System (5) requires initial conditions for orbit calculation. In accelerator physics a particle is considered to come from $z=-\infty$ and it is convenient to determine initial conditions such that the particle orbit would match a drift with given parameters $x_{0}, x_{p 0} \equiv d x / d z, y_{0}, y_{p 0} \equiv$ $d y / d z$ at some abstract entrance plane of the solenoid located at $z=0$ :

$$
\mathbf{r}(z)_{B_{0}=0}=\mathbf{r}_{0}+\mathbf{r}_{p 0} z=\left(\begin{array}{c}
x(z) \\
y(z)
\end{array}\right)_{B_{0}=0}=\left(\begin{array}{l}
x_{0}+x_{p 0} z \\
y_{0}+y_{p 0} z
\end{array}\right)
$$

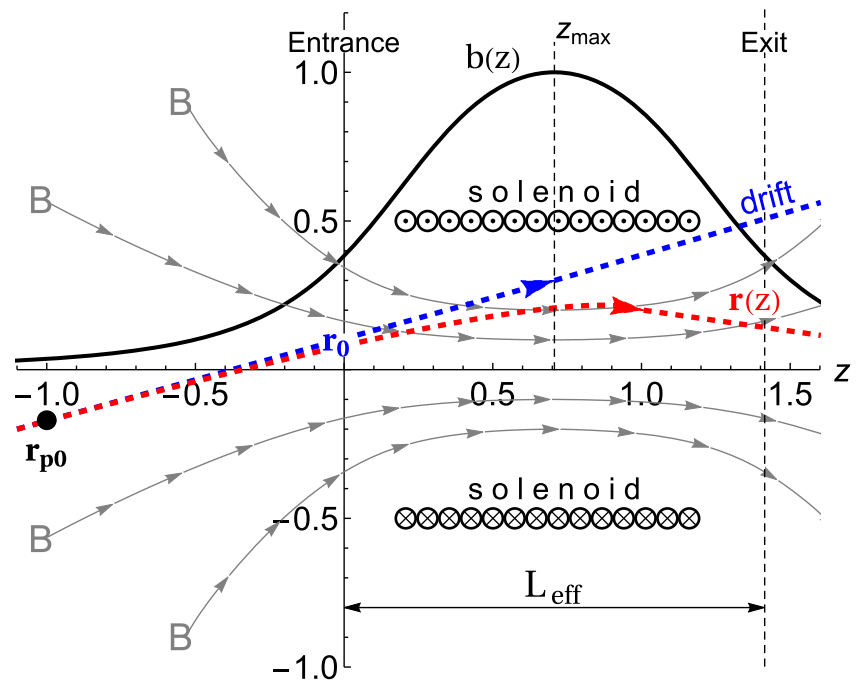

FIG. 1. Schematic of particle dynamics in magnetic field of solenoid. The blue dashed line represents an abstract drift of particle coming from $z=-\infty$ in the case of zero magnetic field [see Eq. (6)]. The red dashed curve represents particle orbit $\mathbf{r}(\mathbf{z})$ in solenoidal magnetic field. The gray arrow lines represent the magnetic field distribution in the solenoid. The vertical axis represents relative scale for all of schematics listed here.

After that we obtain an integrodifferential equations from (5) that contain the initial conditions (6):

$$
\begin{aligned}
x(z)= & x_{0}+x_{p 0} z+k \int_{-\infty}^{z} \int_{-\infty}^{z}\left[b \frac{d y}{d z}+\frac{y}{2} \frac{d b}{d z}\right. \\
& \left.-\frac{x^{2}+y^{2}}{16}\left(y \frac{d^{3} b}{d z^{3}}+4 \frac{d y}{d z} \frac{d^{2} b}{d z^{2}}\right)\right] d z d z \\
y(z)= & y_{0}+y_{p 0} z-k \int_{-\infty}^{z} \int_{-\infty}^{z}\left[b \frac{d x}{d z}+\frac{x}{2} \frac{d b}{d z}\right. \\
& \left.-\frac{x^{2}+y^{2}}{16}\left(x \frac{d^{3} b}{d z^{3}}+4 \frac{d x}{d z} \frac{d^{2} b}{d z^{2}}\right)\right] d z d z .
\end{aligned}
$$

The system (7) can be solved in form of a series expansion of $x(z)$ and $y(z)$ in powers of the $k$ parameter:

$$
x(z)=\sum_{n=0}^{\infty} x_{n}(z) k^{n} \quad y(z)=\sum_{n=0}^{\infty} y_{n}(z) k^{n} .
$$

After substituting (8) into (7) and equating terms with equal powers of $k$ we obtain explicit recurrence formulas for $n$th series terms $x_{n}(z)$ and $y_{n}(z)$ on the left side that depend on lower order terms $i=0 \ldots n-1$ on the right side of the expression: 


$$
\begin{aligned}
& x_{n}(z)=+\int_{-\infty}^{z} \int_{-\infty}^{z}\left[b \frac{d y_{n-1}}{d z}+\frac{y_{n-1}}{2} \frac{d b}{d z}-\sum_{i=0}^{n-1} \sum_{j=0}^{i} \frac{x_{j} x_{i-j}+y_{j} y_{i-j}}{16}\left(x_{n-i-1} \frac{d^{3} b}{d z^{3}}+4 \frac{d x_{n-i-1}}{d z} \frac{d^{2} b}{d z^{2}}\right)\right] d z d z \\
& y_{n}(z)=-\int_{-\infty}^{z} \int_{-\infty}^{z}\left[b \frac{d x_{n-1}}{d z}+\frac{x_{n-1}}{2} \frac{d b}{d z}-\sum_{i=0}^{n-1} \sum_{j=0}^{i} \frac{x_{j} x_{i-j}+y_{j} y_{i-j}}{16}\left(y_{n-i-1} \frac{d^{3} b}{d z^{3}}+4 \frac{d y_{n-i-1}}{d z} \frac{d^{2} b}{d z^{2}}\right)\right] d z d z .
\end{aligned}
$$

The formulas (9) have an explicit form that suggests an iterative algorithm that we chosen in this paper. We use Cartesian coordinates for the sake of simplicity. For example, a cylindrical system of coordinates yields a similar recurrence equation with $r_{n}(z)$ in both the left and the right side, but more complicated calculations are required to extract it from there. Terms $x_{0}(z), y_{0}(z)$ are trivial drift solution (6) and $x_{1}(z), y_{1}(z)$ are calculated by substituting $x_{0}(z), y_{0}(z)$ into (7) and integrating twice. The first order solution in $k$ for the particle orbit and its slope is:

$$
\begin{aligned}
x(z)= & x+x_{p} z+\frac{k}{16}\left[4 x_{p}\left(x_{p} y-y_{p} x\right) \int_{-\infty}^{z} z b^{\prime} d z-b^{\prime}\left(y+y_{p} z\right)\left(x+x_{p} z\right)^{2}-b^{\prime}\left(y+y_{p} z\right)^{3}+2 b y_{p}\left(y^{2}-x^{2}+4 z^{2}\right)\right. \\
& \left.+2 b y z\left(4-x_{p}^{2}+y_{p}^{2}\right)+2\left(y+y_{p} z\right)\left(2 b x x_{p}-\left(4-x_{p}^{2}-y_{p}^{2}\right) \int_{-\infty}^{z} z b^{\prime} d z\right)\right] \\
x_{p}(z)= & \frac{d x(z)}{d z}
\end{aligned}
$$

And there are similar expressions for $y(z), y_{p}(z)$. Here we write $\left\{x, x_{p}, y, y_{p}\right\} \equiv\left\{x_{0}, x_{p 0}, y_{0}, y_{p 0}\right\}$ for simplicity. For higher orders the expansion solution (10) becomes cumbersome and practically unusable so it needs to be transformed into the form of a transfer map.

\section{TRANSFER MAPS}

Accelerator physics calculates single-particle beam dynamics mostly by using transfer map $\mathbf{M}$ that sends initial phase space coordinates $\overrightarrow{\mathbf{z}}_{i}$ into final coordinates $\overrightarrow{\mathbf{z}}_{f}=\mathbf{M}\left(\overrightarrow{\mathbf{z}}_{i}\right)$ without using $z$ or time-dependent orbit formalism. The linear transfer map for a solenoid written in matrix form can be found for example in $[1,15]$ :

$$
\mathbf{M}_{\text {solenoid }}=\mathbf{M}_{\text {exit }} \mathbf{M}_{\text {body }} \mathbf{M}_{\text {entrance }}=\left(\begin{array}{cccc}
1 & 0 & 0 & 0 \\
0 & 1 & -\frac{k}{2} & 0 \\
0 & 0 & 1 & 0 \\
\frac{k}{2} & 0 & 0 & 1
\end{array}\right)\left(\begin{array}{cccc}
1 & \frac{\sin k L}{k} & 0 & \frac{1-\cos k L}{k} \\
0 & \cos k L & 0 & \sin k L \\
0 & -\frac{1-\cos k L}{k} & 1 & \frac{\sin k L}{k} \\
0 & -\sin k L & 0 & \cos k L
\end{array}\right)\left(\begin{array}{cccc}
1 & 0 & 0 & 0 \\
0 & 1 & \frac{k}{2} & 0 \\
0 & 0 & 1 & 0 \\
-\frac{k}{2} & 0 & 0 & 1
\end{array}\right)
$$

This map formally consists of three parts (see Fig. 1): an entrance fringe field $\mathbf{M}_{\text {entrance }}$ located at $z=0, \mathbf{M}_{\text {body }}$ that represents the exact analytic solution of particle motion in the longitudinal magnetic field $B_{0}$ between $0<z<L$ and an exit fringe field $\mathbf{M}_{\text {exit }}$ located at $z=L$. Particles drift in free space through drift transfer maps before and after the map.

\section{A. Entrance transfer map}

In this section we will find transfer map for "halfsolenoid" from $z=-\infty$ to $z=z_{\max }$ in the following form of sequential maps:

$$
\mathbf{S}_{\text {entrance }}=\mathbf{K}_{\text {kick }} \mathbf{M}_{\text {body }} \mathbf{T}_{\text {entrance }} \mathbf{D}_{\text {drift }}
$$

After that, we can easily write the map for the other half of solenoid from $z=z_{\max }$ to $z=\infty$ using symmetry properties. In Eq. (12) we basically need to find a so called "fringe field" map $\mathbf{T}_{\text {entrance }}$ because all other maps are considered to be known: $\mathbf{D}_{\text {drift }}$ is the linear drift map [1,15] that transfer particle with initial conditions (6) from $z=-\infty$ to $z=0$ and finishes with coordinates $\left\{x_{0}, x_{p 0}, y_{0}, y_{p 0}\right\}$ at the entrance of the thin element $\mathbf{T}_{\text {entrance }} . \mathbf{M}_{\text {body }}$ is the linear transfer matrix (11) but with parameter of length $L$ replaced by $z_{\max }$ has not been defined yet. $\mathbf{K}_{\text {kick }}$ is a thin nonlinear transfer map at the center of the solenoid $z_{\max }$ that we define as:

$\mathbf{K}_{\mathrm{kick}} \triangleq\left(\begin{array}{c}x^{f} \\ x_{p}^{f} \\ y^{f} \\ y_{p}^{f}\end{array}\right)=\left(\begin{array}{c}x \\ x_{p}-\frac{k}{16} y\left(x^{2}+y^{2}\right) b^{\prime \prime}\left(z_{\max }\right) \\ y \\ y_{p}+\frac{k}{16} x\left(x^{2}+y^{2}\right) b^{\prime \prime}\left(z_{\max }\right)\end{array}\right)$

Formally, it is possible to define any kind of transfer map construction (12) for example without $\mathbf{K}_{\text {kick }}$ but the ultimate goal is to replace the orbit formalism (10) in such 
a way that the map sequence (12) and especially $\mathbf{T}_{\text {entrance }}$ is as simple as possible. Now, to find the transfer map, we equate the final coordinates of both formalisms (12), (10) at the center of solenoid:

$$
\left(\begin{array}{c}
x\left(z_{\max }\right) \\
x_{p}\left(z_{\max }\right) \\
y\left(z_{\max }\right) \\
y_{p}\left(z_{\max }\right)
\end{array}\right)=\mathbf{K}_{\mathrm{kick}} \mathbf{M}_{\text {body }} \mathbf{T}_{\text {entrance }}(\overrightarrow{\mathbf{z}})
$$

This gives us an equation for the $\mathbf{T}_{\text {entrance }}$ map. Here, $\overrightarrow{\mathbf{z}}$ denotes input coordinates $\left\{x, x_{p}, y, y_{p}\right\}$ that are equal to $\left\{x_{0}, x_{p 0}, y_{0}, y_{p 0}\right\}$ from initial condition (6). The solution for $\mathbf{T}_{\text {entrance }}$ can be written out in a general form:

$$
\mathbf{T}_{\text {entrance }}(\overrightarrow{\mathbf{z}})=\left(\mathbf{K}_{\text {kick }} \mathbf{M}_{\text {body }}\right)^{-1}\left(\begin{array}{c}
x\left(z_{\text {max }}\right) \\
x_{p}\left(z_{\text {max }}\right) \\
y\left(z_{\text {max }}\right) \\
y_{p}\left(z_{\text {max }}\right)
\end{array}\right)
$$

By substituting expressions (13), (11) and (10) into (15) and taking into account that $b\left(z_{\max }\right)=1$ and $b^{\prime}\left(z_{\max }\right)=0$ we obtain an explicit form of the transfer map (15). Also we can define the integrals in (10) to be zero for simplicity:

$$
\int_{-\infty}^{z_{\max }} z b^{\prime}(z) d z=0
$$

This will automatically define position of the center of solenoid $z_{\max }$ relative to the entrance fringe field that we placed in $z=0$ by the definition:

$$
z_{\max }=\int_{-\infty}^{z_{\max }} b(z) d z
$$

For calculation of (15), because we used only the first order map (10) in $k$ we also need to truncate resulting expression (15) to the first order in $k$ and the resulting map is

$$
\left(\begin{array}{c}
x^{f} \\
x_{p}^{f} \\
y^{f} \\
y_{p}^{f}
\end{array}\right)=\left(\begin{array}{c}
x+\frac{k}{8}\left(2 x y x_{p}+y_{p}\left(y^{2}-x^{2}\right)\right) \\
x_{p}+\frac{k}{8}\left(4 y+2 x_{p} y_{p} x+y\left(y_{p}^{2}-x_{p}^{2}\right)\right) \\
y-\frac{k}{8}\left(2 x y y_{p}+x_{p}\left(x^{2}-y^{2}\right)\right) \\
y_{p}-\frac{k}{8}\left(4 x+2 x_{p} y_{p} y+x\left(x_{p}^{2}-y_{p}^{2}\right)\right)
\end{array}\right) \text {. }
$$

The transfer map for higher orders in $k$ can be calculated by calculating the corresponding higher order orbit (10). The first order map (18) agrees with the first order nonlinear map calculated by the Lie methods through integration of Hamiltonian [16]. Detailed consideration of the transformation (15) shows that (18) can be obtained directly from (10) by using the rules $b=1, b^{\prime}=0, b^{\prime \prime}=0$ and the integrals (16) must be equal to zero. Also all $z$-polynomial terms of the form $F(z) z^{i}$ or $c z^{i}$ must be equal to zero.

\section{B. Exit transfer map}

After tracking through the entrance half of solenoid by (12), a particle moves through the exit half of solenoid from $z=z_{\max }$ to $z=+\infty$. If the magnetic field profile $b(z)$ is symmetric, i.e., $b\left(z_{\max }+\Delta z\right)=b\left(z_{\max }-\Delta z\right)$ for any $\Delta z$ then we can immediately write out the transfer map for the exit half using the property of T-symmetry in classical mechanics:

$$
\begin{aligned}
\mathbf{S}_{\text {exit }} & =\mathbf{I S}_{\text {entrance }}^{-1} \mathbf{I} \\
\mathbf{I} & =\left(\begin{array}{cccc}
-1 & 0 & 0 & 0 \\
0 & 1 & 0 & 0 \\
0 & 0 & 1 & 0 \\
0 & 0 & 0 & -1
\end{array}\right)
\end{aligned}
$$

that basically amounts to finding the inverse map of $\mathbf{S}_{\text {entrance. Substituting (12) into (19) and after performing }}$ operator transformations we get the exit transfer map from $z=z_{\max }$ to $z=+\infty$ :

$$
\mathbf{S}_{\text {exit }}=\mathbf{D}_{\text {drift }} \mathbf{T}_{\text {exit }} \mathbf{M}_{\text {body }} \mathbf{K}_{\text {kick }}^{-1}
$$

$\mathbf{T}_{\text {exit }}$ is found by using the transformation (19):

$$
\mathbf{T}_{\text {exit }}=\mathbf{I T}_{\text {entrance }}^{-1} \mathbf{I}
$$

because all other transfer maps of (20) are known.

\section{Full solenoid transfer map}

The maps (12) and (20) can be applied sequentially if its needed to get particle phase space parameters at the center of the solenoid $z_{\max }$. However, it is usually necessary to track particle through the whole solenoid. In this case the total transfer map of solenoid will be

$$
\mathbf{S}_{\text {total }}=\mathbf{S}_{\text {exit }} \mathbf{S}_{\text {entrance }}=\mathbf{T}_{\text {exit }} \mathbf{M}_{\text {body }} \mathbf{T}_{\text {entrance }}
$$

and is presented here without drifts. $\mathbf{M}_{\text {body }}$ is the matrix (11) with parameter $L=2 z_{\max }$ or

$$
L=L_{\mathrm{eff}}=\int_{-\infty}^{+\infty} b(z) d z=\frac{1}{B_{0}} \int_{-\infty}^{+\infty} B(z) d z
$$

which is the effective length of the solenoid (see Fig. 1). Here we recall again that $k$ is defined by (5) through the amplitude of magnetic field $B_{0}=B\left(z_{\max }\right)$.

\section{LEADING ORDER TRANSFER MAP}

The transfer map (18) for higher order expansions in the $k$-parameter is much simpler than (10) but still very cumbersome and not symplectic. By using the recurrence formula (9) we can analyze higher orders terms deriving and keeping only so-called leading order $b(z)^{n}$ terms in (10) that gives unit $b(z)^{n} \rightarrow 1$ in the resulting transfer map. After transformations (9) we will get a recurrence formula for the $n$th order expansion of the transfer map that will contain a pure dependence on input parameters $\left\{x, x_{p}, y, y_{p}\right\}$ because $b(z)=1$ : 


$$
\begin{aligned}
x_{n}= & \frac{1}{16 n} \sum_{i=0}^{n-2} \sum_{j=0}^{i} x_{n-i-2}\left(x_{j} x_{i-j}+y_{j} y_{i-j}\right)+\frac{1}{8 n} \sum_{i=0}^{n-1} \sum_{j=0}^{i}\left(y_{n-i-1}^{\prime}\left(y_{j} y_{i-j}-x_{j} x_{i-j}\right)+2 x_{n-i-1}^{\prime} x_{j} y_{i-j}\right) \\
x_{n}^{\prime}= & +\left(1-\frac{1}{2 n}\right) y_{n-1}-\frac{1}{16 n} \sum_{i=0}^{n-2} \sum_{j=0}^{i}\left(x_{n-i-2}^{\prime}\left(3 x_{j} x_{i-j}+y_{j} y_{i-j}\right)+2 y_{n-i-2}^{\prime} x_{j} y_{i-j}\right) \\
& +\frac{1}{8 n} \sum_{i=0}^{n-1} \sum_{j=0}^{i}\left(y_{n-i-1}\left(y_{j}^{\prime} y_{i-j}^{\prime}-x_{j}^{\prime} x_{i-j}^{\prime}\right)+2 x_{n-i-1} x_{j}^{\prime} y_{i-j}^{\prime}\right) \\
y_{n}= & \frac{1}{16 n} \sum_{i=0}^{n-2} \sum_{j=0}^{i} y_{n-i-2}\left(x_{j} x_{i-j}+y_{j} y_{i-j}\right)+\frac{1}{8 n} \sum_{i=0}^{n-1} \sum_{j=0}^{i}\left(x_{n-i-1}^{\prime}\left(y_{j} y_{i-j}-x_{j} x_{i-j}\right)-2 y_{n-i-1}^{\prime} x_{j} y_{i-j}\right) \\
y_{n}^{\prime}= & -\left(1-\frac{1}{2 n}\right) x_{n-1}-\frac{1}{16 n} \sum_{i=0}^{n-2} \sum_{j=0}^{i}\left(y_{n-i-2}^{\prime}\left(3 y_{j} y_{i-j}+x_{j} x_{i-j}\right)+2 x_{n-i-2}^{\prime} x_{j} y_{i-j}\right) \\
& +\frac{1}{8 n} \sum_{i=0}^{n-1} \sum_{j=0}^{i}\left(x_{n-i-1}\left(y_{j}^{\prime} y_{i-j}^{\prime}-x_{j}^{\prime} x_{i-j}^{\prime}\right)-2 y_{n-i-1} x_{j}^{\prime} y_{i-j}^{\prime}\right)
\end{aligned}
$$

Finally, to calculate the leading order transfer map for $\mathbf{T}_{\text {entrance }}$ we need to calculate infinite series:

$$
\mathbf{T}_{\text {entrance }} \triangleq\left(\begin{array}{c}
x^{f} \\
x_{p}^{f} \\
y^{f} \\
y_{p}^{f}
\end{array}\right)=\left(\begin{array}{c}
\sum_{n=0}^{\infty} x_{n} k^{n} \\
\sum_{n=0}^{\infty} x_{n}^{\prime} k^{n} \\
\sum_{n=0}^{\infty} y_{n} k^{n} \\
\sum_{n=0}^{\infty} y_{n}^{\prime} k^{n}
\end{array}\right)
$$

We skip all intermediate calculations in this paper and present solution that can be verified by (24) after the Taylor series expansion in $k$ :

$$
\begin{aligned}
\mathbf{T}_{\text {entrance }} \triangleq\left(\begin{array}{c}
x^{f} \\
x_{p}^{f} \\
y^{f} \\
y_{p}^{f}
\end{array}\right)=\left(\begin{array}{c}
R \cos \Phi \\
\frac{Q}{R} \cos \Phi-\left(\frac{J}{R}-\frac{k R}{2}\right) \sin \Phi \\
R \sin \Phi \\
\frac{Q}{R} \sin \Phi+\left(\frac{J}{R}-\frac{k R}{2}\right) \cos \Phi
\end{array}\right) \\
\Phi=\phi-Q\left[\frac{k}{8}-\frac{R^{2}}{J^{3}}\left(4\left(1-e^{\frac{k J}{4}}\right)+k J\left(1+\frac{k J}{8}\right)\right)\right] \\
R=\sqrt{\frac{J^{2}}{4+k J+e^{\frac{k J}{4}}\left(\frac{J^{2}}{r^{2}}-4\right)}} \\
Q=q\left(1-\left(4-e^{\left.-\frac{k J}{4}(4+k J)\right)} \frac{r^{2}}{J^{2}}\right)\right. \\
\phi=\arctan (x, y) \\
r=\sqrt{x^{2}+y^{2}} \\
q=x x_{p}+y y_{p} \\
J=x y_{p}-y x_{p} .
\end{aligned}
$$

The fringe field map (26) is sometimes called the hardedge model because it depends only on the amplitude of magnetic field $B_{0}=\int B^{\prime}(z) d z$ in form of $k$ parameter without considering effect of the more detailed magnetic field shape $B(z)$. The exit fringe field can be calculated in analytic form by using (21) but we will do it in the next section for a more advanced transfer map.

\section{SOFT-EDGE TRANSFER MAP}

Apparently, the leading order terms $b(z)$ impact only the nonlinear effect because the transfer map (26) turns into (11): $T_{\text {entrance }} \rightarrow M_{\text {entrance }}$ when taking the linear part of its input parameters $\left\{x, x_{p}, y, y_{p}\right\}$. Calculating the second and higher order transfer map (18) reveals many different coefficients of the following type:

$$
\begin{aligned}
& m_{i}=\int_{-\infty}^{z_{\max }} z^{i} b(z) b^{\prime}(z) d z \\
& p_{i}=\int_{-\infty}^{z_{\max }} z^{i} b^{\prime}(z)^{2} d z \\
& s_{i}=\int_{-\infty}^{z_{\max }} z^{i} b^{\prime \prime}(z)^{2} d z \\
& b(z)=\frac{B(z)}{B_{\max }}
\end{aligned}
$$

Recall again that the frame of integration (27) satisfies conditions (16), (17). The parameter $m_{0}=b\left(z_{\max }\right)^{2} / 2=$ $1 / 2$ is one of the hard-edge terms that has been taken into account in the previous section. In this paper we will take into account only four soft-edge coefficients: $m_{1}, m_{2}, p_{0}, s_{0}$ that is sufficient to derive an accurate solution of the original equation (5) that we are solving. Generally, the 
error due to magnetic field truncation (3), (5) is much larger than the error due to the limited system of the soft-edge parameters.

The soft-edge transfer map can be calculated exactly like the leading order map in the previous section. In this section we skip all intermediate transformations and calculations and present final solution (25) for the entrance transfer map depending on $m_{1}, m_{2}, p_{0}, s_{0}$ :

$$
\begin{aligned}
\mathbf{T}_{\text {entrance }} \triangleq & \left(\begin{array}{c}
x^{f} \\
x_{p}^{f} \\
y^{f} \\
y_{p}^{f}
\end{array}\right)=\left(\begin{array}{c}
R \cos \Phi \\
\frac{Q}{R} \cos \Phi-\left(\frac{J}{R}-\frac{k R}{2}\right) \sin \Phi \\
R \sin \Phi \\
\frac{Q}{R} \sin \Phi+\left(\frac{J}{R}-\frac{k R}{2}\right) \cos \Phi
\end{array}\right) \\
\Phi= & \phi-Q\left[\frac{k}{8}-\frac{R^{2}}{J^{3}}\left(4\left(1-e^{\frac{k J}{4}}\right)+k J\left(1+\frac{k J}{8}\right)\right)\right] \\
R= & \sqrt{\frac{J^{2}}{4+k J+e^{\frac{k J}{4}}\left(\frac{J^{2}}{r^{2}} e^{\frac{k^{2} m_{2}}{2}}-4\right)}} \\
Q= & \left(1-\left(4-e^{-\frac{k J}{4}}(4+k J)\right) \frac{r^{2}}{J^{2}} e^{-\frac{k^{2} m_{2}}{2}}\right) \\
& \times\left(r e^{-\frac{k^{2} m_{2}}{4}} \sqrt{\frac{2}{3}} \tan \left[\sqrt{\frac{3}{8}} k^{2} m_{1} r e^{-\frac{k^{2} m_{2}}{4}}\right]\right. \\
& \left.-\frac{k^{2}}{8} r^{4} p_{0}-\frac{3 k^{2}}{256} r^{6} s_{0}+q\right) \\
\phi= & \arctan (x, y) \\
r= & \sqrt{x^{2}+y^{2}} \\
q= & x x_{p}+y y_{p} \\
J= & x y_{p}-y x_{p}
\end{aligned}
$$

Exit map for symmetric solenoids $b\left(z_{\max }+\Delta z\right)=$ $b\left(z_{\max }-\Delta z\right)$ can be derived directly from (28) by (21):

$$
\begin{aligned}
\mathbf{T}_{\text {exit }} \triangleq & \left(\begin{array}{c}
x^{f} \\
x_{p}^{f} \\
y^{f} \\
y_{p}^{f}
\end{array}\right)=\left(\begin{array}{c}
R \cos \Phi \\
\frac{Q}{R} \cos \Phi-\frac{J}{R} \sin \Phi \\
R \sin \Phi \\
\frac{Q}{R} \sin \Phi+\frac{J}{R} \cos \Phi
\end{array}\right) \\
\Phi= & \phi+q\left[\frac{k}{8}-\frac{r^{2}}{J^{3}}\left(4\left(1-e^{\frac{k J}{4}}\right)+k J\left(1+\frac{k J}{8}\right)\right)\right] \\
R= & e^{\frac{k^{2} m_{2}}{4}} \sqrt{\frac{J^{2}}{4+e^{-\frac{k J}{4}}\left(\frac{J^{2}}{r^{2}}-4-k J\right)}} \\
Q= & q\left(1-\left(4-e^{-\frac{k J}{4}}(4+k J)\right) \frac{R^{2}}{J^{2}} e^{-\frac{k^{2} m_{2}}{2}}\right)^{-1} \\
& +R e^{-\frac{k^{2} m_{2}}{4}} \sqrt{\frac{2}{3}} \tan \left[\sqrt{\left.\frac{3}{8} k^{2} m_{1} R e^{-\frac{k^{2} m_{2}}{4}}\right]}\right. \\
& -\frac{k^{2}}{8} R^{4} p_{0}-\frac{3 k^{2}}{256} R^{6} s_{0} \\
\phi= & \arctan (x, y) \\
r= & \sqrt{x^{2}+y^{2}} \\
q= & x x_{p}+y y_{p} \\
J= & x y_{p}-y x_{p}+\frac{k}{2}\left(x^{2}+y^{2}\right) .
\end{aligned}
$$

For symmetric $B(z)$ fields all coefficients $m_{1}, m_{2}, p_{0}, s_{0}$ in (28), (29) are the same with the same sign. If the field $B_{n s}(z)$ is not symmetric then coefficients for the entrance fringe field (28) must be calculated as in (27) by using $B_{n s}(z)$, and for the exiting field by using its mirror transformation relative to the $z_{\max }$ point and integrating (27) as usual from $-\infty$ to $z_{\max }$ where $z_{\max }=$ $B_{0}^{-1} \int_{z_{\max }}^{+\infty} B_{n s}(z) d z$ will have slightly different value than (17).

\section{A. Linear transfer map for solenoid}

We will write out soft-edge linear transfer map for solenoid by calculating linear coefficients (28) and (29) for $\left\{x, x_{p}, y, y_{p}\right\}$ phase space coordinates:

$$
\begin{aligned}
\mathbf{M}_{\text {solenoid }} & =\mathbf{M}_{\text {exit }} \mathbf{M}_{\text {body }} \mathbf{M}_{\text {entrance }} \\
& =\left(\begin{array}{cccc}
e^{\frac{k^{2} m_{2}}{4}} & 0 & 0 & 0 \\
\frac{k^{2} m_{1}}{2} e^{-\frac{k^{2} m_{2}}{4}} & e^{-\frac{k^{2} m_{2}}{4}} & -\frac{k}{2} e^{-\frac{k^{2} m_{2}}{4}} & 0 \\
0 & 0 & e^{\frac{k^{2} m_{2}}{4}} & 0 \\
\frac{k}{2} e^{-\frac{k^{2} m_{2}}{4}} & 0 & \frac{k^{2} m_{1}}{2} e^{-\frac{k^{2} m_{2}}{4}} & e^{-\frac{k^{2} m_{2}}{4}}
\end{array}\right)\left(\begin{array}{cccc}
1 & \frac{\sin k L}{k} & 0 & \frac{1-\cos k L}{k} \\
0 & \cos k L & 0 & \sin k L \\
0 & -\frac{1-\cos k L}{k} & 1 & \frac{\sin k L}{k} \\
0 & -\sin k L & 0 & \cos k L
\end{array}\right)\left(\begin{array}{cccc}
e^{-\frac{k^{2} m_{2}}{4}} & 0 & 0 & 0 \\
\frac{k^{2} m_{1}}{2} e^{-\frac{k^{2} m_{2}}{4}} e^{\frac{k^{2} m_{2}}{4}} & \frac{k}{2} e^{-\frac{k^{2} m_{2}}{4}} & 0 \\
0 & 0 & e^{-\frac{k^{2} m_{2}}{4}} & 0 \\
-\frac{k}{2} e^{-\frac{k^{2} m_{2}}{4}} & 0 & \frac{k^{2} m_{1}}{2} e^{-\frac{k^{2} m_{2}}{4}} & e^{\frac{k^{2} m_{2}}{4}}
\end{array}\right)
\end{aligned}
$$


All zero elements in the fringe field matrices (30) really depend on the $m_{3}$ and $m_{4}$ coefficients that we did not take into account because of its small linear effect.

\section{PROPERTIES OF TRANSFER MAPS}

a. Symplecticity. The nonlinear and linear maps of the solenoid are symplectic [1] analytically in canonical coordinates. Transverse canonical coordinates depend on transverse vector potential $A_{x}$ and $A_{y}$ are equal to zero outside of the solenoid. In this case input and output canonical coordinates of the full solenoid (22) are equal to normal ones $\left\{x, x_{p}, y, y_{p}\right\},\left\{x^{f}, x_{p}^{f}, y^{f}, y_{p}^{f}\right\}$ and the nonlinear (22), (28), (29) or linear (30) maps of the full solenoid are symplectic:

$$
\mathbf{M}^{\top} \mathbf{J M}=\mathbf{J}
$$

where $\mathbf{M}$ is Jacobian of the map and $\mathbf{J}$ is the unit symplectic matrix [1]:

$$
\begin{aligned}
\mathbf{M} & =\frac{\partial\left(x^{f}, x_{p}^{f}, y^{f}, y_{p}^{f}\right)}{\partial\left(x, x_{p}, y, y_{p}\right)} \\
\mathbf{J} & =\left(\begin{array}{cccc}
0 & 1 & 0 & 0 \\
-1 & 0 & 0 & 0 \\
0 & 0 & 0 & 1 \\
0 & 0 & -1 & 0
\end{array}\right)
\end{aligned}
$$

Individual maps $\mathbf{T}_{\text {entrance}}$, or $\mathbf{M}_{\text {entrance }}$ are not symplectic in normal coordinates but symplectic in canonical coordinates because of nonzero transverse vector potential inside of solenoid. When the final coordinates in the Jacobian matrix $\mathbf{M}$ are replaced by canonical ones:

$$
\mathbf{M}=\frac{\partial\left(x^{f}, x_{p}^{f}-k \frac{y^{f}}{2}, y^{f}, y_{p}^{f}+k \frac{x^{f}}{2}\right)}{\partial\left(x, x_{p}, y, y_{p}\right)}
$$

the individual maps $\mathbf{T}_{\text {entrance }}$ and $\mathbf{M}_{\text {entrance }}$ become symplectic (31).

b. Canonical angular momentum is a constant of motion that is equal to:

$$
\begin{aligned}
\text { const }= & x(z) y^{\prime}(z)-y(z) x^{\prime}(z)+\frac{k}{2}\left(x(z)^{2}+y(z)^{2}\right) b(z) \\
& -\frac{k}{16}\left(x(z)^{2}+y(z)^{2}\right)^{2} b^{\prime \prime}(z)
\end{aligned}
$$

in terms of the equation of motion (5) with given truncated magnetic field. Substituting final/output coordinates of (12) into (34) with $z=z_{\max }$ confirms conservation of canonical angular momentum:

$$
\begin{aligned}
& x^{f} y_{p}^{f}-y^{f} x_{p}^{f}+\frac{k}{2}\left(x^{f 2}+y^{f 2}\right)-\frac{k}{16}\left(x^{f 2}+y^{f 2}\right)^{2} b^{\prime \prime}\left(z_{\max }\right) \\
& =x y_{p}-y x_{p}
\end{aligned}
$$

where the resulting expression $x y_{p}-y x_{p}$ is the canonical angular momentum for $k=0$ before the particle enters the solenoid.

c. Hard-edge fringe field limit defined as the unit step function for input and output fringe field: $b^{\prime}(z) \rightarrow \delta(z)$ produces divergent integrals $p_{0}, s_{0} \rightarrow \infty$ (27) and hence the transfer maps (28), (29). The same kind of divergence has been found by integration of Hamiltonian in Lie algebra method [11]. Therefore the nonlinear beam dynamics of solenoid depends on fringe field shape that cannot be simplified by the hard-edge fringe field model.

$d$. Paraxial limitation. The expression under the square root in (28) must be much greater than zero for the paraxial motion approximation to be valid. If it is less than zero then particle will be reflected back by the entrance fringe field of solenoid. This can be demonstrated by solving the full 3D equation of motion (1). This can happen for example at small particle energy or in very strong magnetic fields $k^{2}\left(x^{2}+y^{2}\right)>8$ even if $x_{p}=y_{p}=0$. This effect is called a magnetic mirror and must be estimated and taken into account before applying the paraxial model. The expression under the square root for the exit fringe field (29) is always positive. This means that particle will always exit solenoid with any initial conditions if it starts in its center.

\section{BENCHMARK OF PARTICLE OPTICS}

We now perform a benchmark of our transfer maps for proton motion in the magnetic field of a single current loop with $R=10 \mathrm{~cm}$ radius. The current loop is located at the center $z=0$ with maximum magnetic field $B_{0}=1 \mathrm{~T}$ at the center of coordinates. 3D magnetic field of the current loop has a precise analytic solution [17] and shown in Fig. 2. The $B(0,0, z)$-field profile of the current loop has the following form:

$$
B(0,0, z)=B_{0} b(z)=B_{0} \frac{R^{3}}{\left(R^{2}+z^{2}\right)^{\frac{3}{2}}}
$$

The transfer map parameters for the magnetic field (36) are calculated to be the following: 


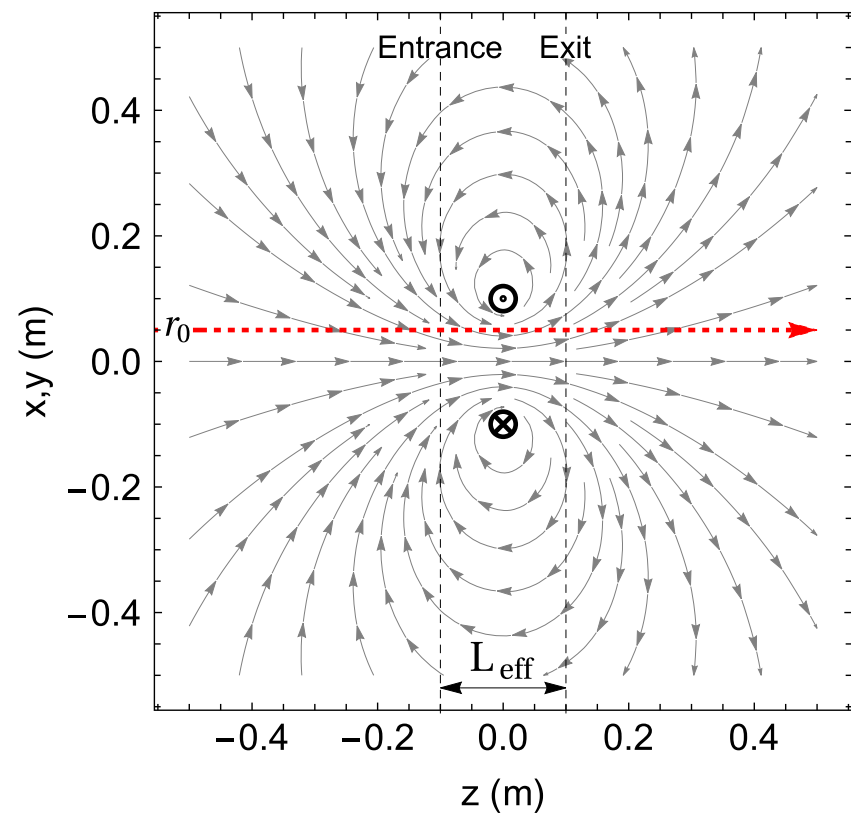

FIG. 2. Simulation of proton dynamics in $3 \mathrm{D}$ field of the current loop with radius $R=0.1 \mathrm{~m}$. The gray arrows show precise magnetic field distribution of the current loop. The proton starts with $\{x, 0, y, 0\}$ parameters where $r_{0}=\sqrt{x^{2}+y^{2}}$.

$$
\begin{aligned}
& z_{\text {entrance }}=-R \\
& z_{\text {exit }}=+R \\
& L_{\text {eff }}=L=2 R \\
& m_{1}=R\left(\frac{1}{2}-\frac{3 \pi}{32}\right) \\
& m_{2}=R^{2}\left(\frac{3}{4}-\frac{3 \pi}{16}\right) \\
& p_{0}=\frac{1}{R} \frac{45 \pi}{256} \\
& s_{0}=\frac{1}{R^{3}} \frac{1575 \pi}{2048} \\
& b^{\prime \prime}\left(z_{\max }\right)=-\frac{3}{R^{2}} .
\end{aligned}
$$

A proton with input parameters $\{x, 0, y, 0\}$ will be focused by the magnetic field and will cross the $z$-axis at some point $z=z_{f}$ called the focal length. By applying the linear model (30) we can calculate the focal length as:

$$
\begin{aligned}
z_{f} & =z_{\text {exit }}-\frac{x^{f}}{x_{p}^{f}}=z_{\text {exit }}-\frac{y^{f}}{y_{p}^{f}} \\
& =z_{\text {exit }}-\frac{2 e^{\frac{k^{2} m_{2}}{2}}\left(\cot \left[\frac{k L}{2}\right]+k m_{1}\right)}{k\left(2 k m_{1} \cot \left[\frac{k L}{2}\right]-1+k^{2} m_{1}^{2}\right)} .
\end{aligned}
$$

Note that it does not depend on the input parameter $r_{0}=\sqrt{x^{2}+y^{2}}$ in linear approximation. Table I shows
TABLE I. Benchmark of the focal length for different proton energies $T_{p}$ and different models and simulations.

\begin{tabular}{llllll}
\hline \hline$T_{p}(\mathrm{MeV})$ & $z_{f}^{\mathrm{a}}(\mathrm{m})$ & $z_{f}^{\mathrm{b}}(\mathrm{m})$ & $z_{f}{ }^{\mathrm{c}}(\mathrm{m})$ & $z_{f}^{\mathrm{d}}(\mathrm{m})$ & $z_{f}{ }^{\mathrm{e}}(\mathrm{m})$ \\
\hline 1 & 0.73873 & 0.73873 & 0.44891 & 0.76209 & 0.73670 \\
10 & 7.15776 & 7.15776 & 4.23124 & 7.18318 & 7.15606 \\
100 & 74.7005 & 74.7005 & 44.0173 & 74.7261 & 74.6988 \\
1000 & 1086.73 & 1086.73 & 640.150 & 1086.75 & 1086.72 \\
\hline
\end{tabular}

${ }^{\mathrm{a}} 2 \mathrm{D}$ z-dependent tracking (5) with truncated field.

b $3 \mathrm{D}$ t-dependent tracking (1) in 3D field of current loop [17].

${ }^{\mathrm{c}}$ Model (11) with $L=\int b(z) d z$ and $B_{0}=B_{\max }=1 T$.

${ }^{\mathrm{d}}$ Model (11) with $L=\frac{\left(\int b(z) d z\right)^{2}}{\int b(z)^{2} d z}$ and $B_{0}=B_{\max } \frac{\int b(z)^{2} d z}{\int b(z) d z}$ [18].

${ }^{\mathrm{e}}$ Focal length (38) obtained by the soft-edge linear model (30).

the focal length obtained by different models and simulations. The initial transverse coordinate of the proton $r_{0}=0.1 \mathrm{~mm}$ was chosen to be small for numerical simulations $a$ and $b$ in order to avoid nonlinear aberrations. The linear model (30) agrees best when compared with numerical simulations $a$ and $b$. Larmor rotation angle of solenoid that equals to $\delta \phi=$ $-k L / 2$ is the same for all linear models $(\mathrm{a}-\mathrm{d})$ of Table I. Nonlinear aberrations start at larger $r_{0}$, as is shown in Fig. 3 for the case of protons with $10 \mathrm{MeV}$ energy. The nonlinear map (22), (28), (29) has a perfect agreement with the 2D simulation (5) and a good agreement with the $3 \mathrm{D}$ simulation (1) for $r_{0}<R / 2$. Figure 3 also shows that there is no difference between 2D and 3D case if magnetic field is precisely calculated.

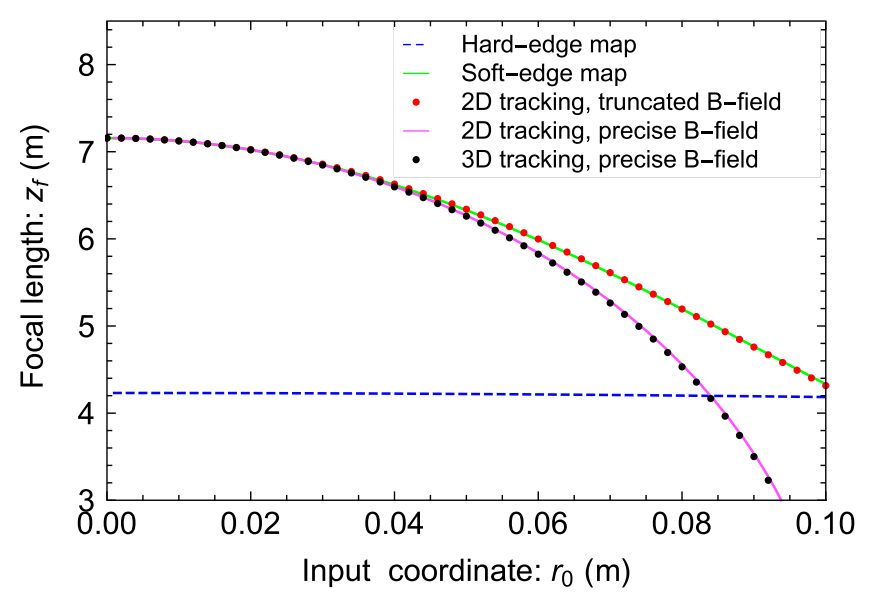

FIG. 3. Focal length as a function of input coordinate $r_{0}=$ $\sqrt{x^{2}+y^{2}}$ for protons with $10 \mathrm{MeV}$ energy. Two lower matching curves show 2D (5) and 3D (1) simulations in case of precisely defined magnetic field of the current loop [17]. Two upper matching curves are for 2D simulations (5) with truncated magnetic field and for the nonlinear soft-edge transfer maps (22), (28), (29) derived from this 2D equation. The single horizontal blue dashed line represents the hardedge model (26) that is equivalent to the soft-edge model with $m_{1}=m_{2}=p_{0}=s_{0}=0$. 
It follows that for more accuracy of the transfer map we need to take more or infinite series expansion (2) for solving $2 \mathrm{D}$ equation (5). The hard edge model represents only $1 \%$ change for the focal length within the solenoid aperture so its not applicable for any aberration estimate.

\section{SUMMARY}

We derived an analytic solution for paraxial charged particle motion in solenoidal magnetic fields that works accurately within the 2D paraxial approximation and for beam sizes up to half the aperture of the solenoid, $r_{\text {beam }}<0.5 R_{\text {solenoid }}$. The accuracy is limited by the precision of the magnetic field given in the form of a truncated series expansion near $z$-axis. For greater accuracy, it is necessary to take the full infinite series expansion of the magnetic field which will be a subject of future works. The method developed in this paper can be extended for other magnets widely used in accelerator physics such as dipoles, quadrupoles, sextupoles etc. Also, it is possible to introduce space charge into the equation of motion and obtain solutions for intense and nonlinear charged-particle beams dynamics in magnetic fields.

\section{ACKNOWLEDGMENTS}

The author acknowledges J. Holmes, S. Cousineau and A. Aleksandrov for the technical review of this work. This manuscript has been authored by UT-Battelle, LLC, under Contract No. DE-AC05-00OR22725 with the U.S. Department of Energy. The United States Government retains, and the publisher, by accepting the article for publication, acknowledges that the United States Government retains a non-exclusive, paid-up, irrevocable, world-wide license to publish or reproduce the published form of this manuscript, or allow others to do so, for United States Government purposes. The Department of Energy will provide public access to these results of federally sponsored research in accordance with the DOE Public Access Plan [19].

[1] A. Chao, K. Mess, M. Tigner, and F. Zimmermann, Handbook of Accelerator Physics and Engineering (World Scientific Publishing, Singapore, 2013).

[2] S. Shanab et al., Superconducting solenoid package design, fabrication, and testing for FRIB, IEEE Trans. Appl. Supercond. 28, 1 (2018).

[3] S. Kim et al., Integration of superconducting solenoids in long cryomodules, in Proceedings of the LINAC2016 Workshop (East Lansing, MI, USA, 2016).
[4] R. Gupta et al., High field HTS solenoid for a muon collider demonstrations, challenges and strategies, IEEE Trans. Appl. Supercond. 24, 1 (2013).

[5] M. A. Green, V. Lebedev, and B. P. Strauss, A superconducting focusing solenoid for the neutrino factory linear accelerator, IEEE Trans. Appl. Supercond. 12, 274 (2002).

[6] S. Nagaitsev et al., Design and simulations of IOTA-a novel concept of integrable optics test accelerator, in Proceedings of the 3rd International Particle Accelerator Conference, New Orleans, LA, 2012 (IEEE, Piscataway, NJ, 2012).

[7] V. Danilov et al., An integrable optics lens for the PSR and SNS, in Proceedings of the 18th Particle Accelerator Conference, New York, 1999 (IEEE, New York, 1999).

[8] J. Holmes, T. Gorlov, N. Evans, M. Plum, and S. Cousineau, Injection of a self-consistent beam with linear space charge force into a ring, Phys. Rev. Accel. Beams 21, 124403 (2018).

[9] K. Makino and M. Bertz, Solenoid elements in COSY INFINITY, in Seventh International Conference on Computational Accelerator Physics (Michigan, USA, 2002).

[10] M. Bertz, Modern Map Methods in Particle Beam Physics (Academic Press, San Diego, 1999).

[11] A. J. Dragt, Lie Methods for Nonlinear Dynamics with Applications to Accelerator Physics (2019), http://www .physics.umd.edu/dsat/.

[12] S. M. Lund, Nonlinear optics in solenoid magnets, in Proceedings of the IPAC2015 Workshop (Richmond, Virginia, 2015).

[13] A. Bondarenko and A. Matveenko, Emittance compensation scheme for the berlinpro injector, in Proceedings of the 2nd International Particle Accelerator Conference, San Sebastiáán, Spain (EPS-AG, Spain, 2011).

[14] H. Matsuda and H. Wollnik, Third order transfer matrices for the fringing field of magnetic and electrostatic quadrupole lenses, Nucl. Instrum. Methods Phys. Res. 103, 177 (1972).

[15] M. Conte and W. M. MacKay, An Introduction to the Physics of Particle Accelerators (World Scientific Publishing, Singapore, 2008).

[16] Y. Cai and Y. Nosochkov, Dynamical effect due to fringe field of the magnet in circular accelerators, in Proceedings of the 21st Particle Accelerator Conference, Knoxville, TN, 2005 (IEEE, Piscataway, NJ, 2005).

[17] J. Simpson, J. Lane, C. Immer, and R. Youngquist, Simple Analytic Expressions for the Magnetic Field of a Circular Current Loop, NASA technical documents Tech. Rep., 2001.

[18] S. Lund, Solenoid focusing, Lecture notes from US Particle Accel. School (2018), https://people.nscl.msu.edu/ lund/ uspas/ap_2018/lec_lund/18.solenoid_ho.pdf.

[19] http://energy.gov/downloads/doe-public-access-plan 